University of Nebraska - Lincoln

DigitalCommons@University of Nebraska - Lincoln

Faculty Publications, Department of Physics and Astronomy

Research Papers in Physics and Astronomy

1976

\title{
H I in Early-Type Galaxies. II. Mass Loss and Galactic Winds
}

S. M. Faber

University of California

J. S. Gallagher

University of Nebraska-Lincoln

Follow this and additional works at: https://digitalcommons.unl.edu/physicsfacpub

Part of the Physics Commons

Faber, S. M. and Gallagher, J. S., "H I in Early-Type Galaxies. II. Mass Loss and Galactic Winds" (1976). Faculty Publications, Department of Physics and Astronomy. 81.

https://digitalcommons.unl.edu/physicsfacpub/81

This Article is brought to you for free and open access by the Research Papers in Physics and Astronomy at DigitalCommons@University of Nebraska - Lincoln. It has been accepted for inclusion in Faculty Publications, Department of Physics and Astronomy by an authorized administrator of DigitalCommons@University of Nebraska Lincoln. 
The Astrophysical Journal, 204:365-378, 1976 March 1

(C) 1976. The American Astronomical Society. All rights reserved. Printed in U.S.A.

\title{
H I IN EARLY-TYPE GALAXIES. II. MASS LOSS AND GALACTIC WINDS*
}

\author{
S. M. FABER \\ Lick Observatory and Board of Studies in Astronomy and Astrophysics, University of California, Santa Cruz \\ AND \\ J. S. Gallagher \\ University of Nebraska-Lincoln, and University of Minnesota, Minneapolis \\ Received 1975 April 21; revised 1975 August 7
}

\begin{abstract}
Implications of observed upper limits to the neutral hydrogen content of $\mathrm{E}$ and $\mathrm{S} 0$ galaxies from Paper I are discussed. If gas shed by dying stars is completely retained by these galaxies and exists as $\mathrm{H}$ I, these upper limits will be exceeded in only $10^{9} \mathrm{yr}$ for E's and S0's and in only $2 \times 10^{8} \mathrm{yr}$ for NGC 4472. Evidently, gas either is hidden in some undetectable form or is removed from the interstellar medium completely.

Several schemes to conceal the mass are proposed, but all seem improbable on one or more grounds. Hence a removal mechanism of some kind seems likely. The most satisfactory candidates for this mechanism appear to be a hot galactic wind (Mathews and Baker 1971) or star formation. Several reasons for favoring the wind hypothesis are presented.

The paper concludes with a more speculative discussion of the possible role of hot winds in spiral galaxies. Because of similarities in stellar population and velocity dispersion between ellipticals and the bulges of spirals, hot winds may operate in spiral bulges as well. Several properties of galaxies along the Hubble sequence might be partially explained on this basis.

In the course of the discussion we summarize several observations which appear to follow logically from the existence of galactic winds: (1) lack of interstellar matter in most early-type galaxies, (2) the existence of young stars and dust in luminous dwarf ellipticals like NGC 205, (3) the fact that radio activity is associated preferentially with massive elliptical galaxies, (4) X-ray sources in clusters of galaxies, and (5) the observed dependence of elliptical metallicity on galaxy
\end{abstract} mass (Larson 1974b).

Subject headings: galaxies: general — interstellar: matter — radio sources: $21 \mathrm{~cm}$ radiation stars: mass loss

\section{INTRODUCTION}

Interstellar material in galaxies plays an important role in galaxy morphology and evolution. The initial mass function and the rate of star formation are determined by conditions in this material, and these two parameters in turn determine the rate of galaxy evolution and the prominence of spiral arms. The interstellar medium is also the reservoir through which heavy elements made by earlier generations of stars are transmitted to later generations. For all these reasons, a knowledge of the physical processes operating in the interstellar medium is essential to our understanding of galaxy evolution. Of particular significance is an understanding of how material is added to the interstellar medium and how it is removed.

Elliptical and S0 galaxies are important to any theory of gas in galaxies simply because they show little evidence of such matter (see, for example, Gallagher et al. 1975, hereafter referred to as Paper I). Either sources of input are negligible, the removal mechanisms are highly efficient, or the material is present in some form that is difficult to detect. Possible injection mechanisms include mass loss from dying stars or the

* Lick Observatory Bulletin, No. 709. infall of intergalactic gas (e.g., Quirk and Tinsley 1973).

We show in this paper that dying stars alone provide sufficient matter so that the first alternative, negligible input, is unlikely. We have also been unable to discover any plausible way of storing mass in the form of an unseen interstellar medium. Hence an efficient removal mechanism of some kind seems probable. The present possibilities appear to be limited to external sweeping, formation of condensed objects, gas ejection by violent nuclear activity, or sweeping by a hot galactic wind. In particular, galactic winds could conceivably operate efficiently in all spheroidal galaxies. We have therefore emphasized this potentially important mechanism throughout our discussion.

If a hot galactic wind can exist in any massive ellipsoidal star system, then this mechanism may also act to reduce the gas content in bulges of later-type spirals. Viewing the Hubble sequence as an unbroken continuum, one would expect the importance of various physical mechanisms to vary smoothly with Hubble type. We hypothesize that winds are a primary removal process in the E galaxies, and that as the bulgeto-disk ratio decreases toward the later spirals, star formation becomes the dominant sink for interstellar 
matter. The paper concludes with speculative remarks on the importance of winds to the origin of S0 galaxies.

The implications of $21 \mathrm{~cm}$ measurements in earlytype galaxies have been discussed previously by Gallagher (1972), Bottinelli et al. (1973), and Knapp and Kerr (1974b), all of whom concluded that the apparent lack of gas in early-type galaxies is surprising. We feel our new data (Paper I) warrant a careful rediscussion of these issues in order to establish whether this lack of gas really poses a difficult contradiction. To this end, we determine the expected mass-injection rate more carefully than has been done in the past and establish a minimum gas content which can reasonably by expected. We then discuss (and reject) a lengthy list of possible storage mechanisms. Although we, too, conclude that the lack of gas is unexpected, we believe that such a careful discussion is necessary to place the subject on a firm foundation.

\section{EXPECTED GAS INJECTION RATES INTO THE INTERSTELLAR MEDIUM IN EARLY-TYPE GALAXIES}

The true injection rate of gas shed by stars into the interstellar medium cannot be observed directly for distant galaxies. It must be inferred from our knowledge of the stellar population, together with observations of mass loss in similar stars in the solar neighborhood and in Local Group galaxies. Because stars in ellipticals could conceivably differ in some significant respect from their counterparts in the solar neighborhood, this inference is necessarily somewhat uncertain. However, planetary nebulae have recently been discovered in considerable numbers in M32, NGC 205, NGC 185, and the bulge in M31 (Ford et al. 1973; Ford, private communication). This observation is of special importance because it confirms directly that mass loss is indeed occurring in these old stellar populations; furthermore, the observed numbers of these planetaries can be used to estimate an empirical lower limit to the mass-loss rate.

The colors and spectra of E and S0 galaxies indicate that virtually all of the light is produced by an old stellar population with a turnoff near $1 M_{\odot}$ and an age near $10^{10}$ yr (Faber 1972; Larson and Tinsley 1974). Abundant evidence suggests that significant mass loss occurs when a star near $1 M_{\odot}$ becomes an $\mathrm{M}$ giant and then a planetary (e.g., Rose and Tinsley 1974). We have adopted this hypothesis in what follows. In this paper, all luminosities are photographic luminosities $L_{\mathrm{pg}}$.

Averaging planetary birthrates scaled-up from observations in the solar neighborhood, the Magellanic Clouds, and M32, Faber (1975) finds a mean birthrate for planetaries of $0.03 \mathrm{yr}^{-1}\left(10^{9} L_{\odot}\right)^{-1}$. Ford and Jenner $(1975)$ find a birthrate $\geq 0.012 \mathrm{yr}^{-1}\left(10^{9} L_{\odot}\right)^{-1}$ in M32. In view of the observational uncertainties, these numbers agree well with the theoretical rate of $0.05 \mathrm{yr}^{-1}\left(10^{9} L_{\odot}\right)^{-1}$ for model $\mathrm{S}$ of Larson and Tinsley (1974) (Tinsley, private communication). Adopting the theoretical value as the more certain, together with a mean mass per planetary of $0.2 M_{\odot}$, we obtain an annual mass-loss rate of $0.010 M_{\odot}$ $\mathrm{yr}^{-1}\left(10^{9} L_{\odot}\right)^{-1}$ from planetaries alone.

Instead of estimating mass loss from Miras directly we resort to more general arguments about the total mass loss per star in the Mira and planetary stages combined. This total is presumably equal to the difference between the turnoff mass and the average mass of white dwarfs. Since Weidemann (1968) finds a mean white dwarf mass of $0.65 M_{\odot}$, we infer a total mass loss per star $\geqslant 0.3 M_{\odot}$.

The energy distributions of ellipticals themselves furnish further evidence that the mass loss per star is $0.3 M_{\odot}$. Rose and Tinsley (1974) find that the integrated colors of ellipticals are closely matched using $\mathbf{M}$ giants having maximum core masses of $0.7 M_{\odot}$. (Giants with core masses of $1.0 M_{\odot}$, on the other hand, lead to colors that are too red.)

For our final adopted mass-loss rate in early-type galaxies, we combine the theoretical evolution rate of $\mathrm{K}$ giants in model $\mathrm{S}$ of Larson and Tinsley with a mean mass loss per star of $0.3 M_{\odot}$. This yields a final ejection rate of $0.015 M_{\odot} \mathrm{yr}^{-1}\left(10^{9} L_{\odot}\right)^{-1}$.

It is desirable though difficult to estimate the uncertainty in the computed mass-loss rate. This uncertainty arises from errors in the mass loss per star (difference between turnoff mass and core mass) and from errors in the evolution rate and numbers of giant stars. The turnoff mass depends upon the match between computed and observed colors for early-type galaxies. Comparison with the computed rates of change of $(B-V)$ for the models of Larson and Tinsley indicates that the estimated age of their models can be off at most by a factor of 1.5 . This means the turnoff mass must lie between 0.9 and $1.1 M_{\odot}$. If the core mass is equated with the mean mass of white dwarfs in the solar neighborhood, the formal error in this quantity is less than $0.1 M_{\odot}$. For early-type galaxies, the two errors in evolutionary rate and numbers of $\mathbf{K}$ giants nearly cancel one another. For example, if the giants evolve more slowly, they are less luminous, and more of them are required to produce the observed galaxy luminosity. For this reason, errors in the evolutionary calculations are not important.

Combining these errors, one finds that the computed mass-loss rate should be correct within a factor of 3 . In $\S$ III and $\S$ IV we show that this formal accuracy in the mass-loss rate leads to several important conclusions concerning gas in early-type galaxies. Those conclusions, it seems, can be avoided only by altering our basic assumptions about mass loss, not by varying the exact parameters we have used.

\section{THEORETICAL INJECTION RATES COMPARED WITH OBSERVATIONAL UPPER LIMITS TO NEUTRAL HYDROGEN MASSES}

If the gas shed from dying stars remains as optically thin neutral atomic hydrogen, a mass-loss rate of $0.015 M_{\odot} \mathrm{yr}^{-1}\left(10^{9} L_{\odot}\right)^{-1}$ should produce a detectable $21 \mathrm{~cm}$ signal in an embarrassingly short time. For 
example, if the upper limits for eight $\mathrm{E}$ and six S0 galaxies given in Paper I (increased by $33 \%$ to allow for helium content) are compared with the mean mass-loss rate calculated using the average galaxy luminosity in each group, predicted gas masses exceed the observed upper limits in only $8.5 \times 10^{8} \mathrm{yr}$ for the ellipticals and in $1.3 \times 10^{9} \mathrm{yr}$ for the S0's. Assuming the new upper limit for NGC 4472 set by Knapp and Kerr (1974b) (see Paper I), one obtains a contradiction for this galaxy in only $1.8 \times 10^{8} \mathrm{yr}$.

In expressing the problem in these terms, we have avoided assumptions about the mean mass-loss rate averaged over the entire lifetime of early-type galaxies. The analytic approximations of Tinsley (1973), for example, indicate that with a Salpeter luminosity function for the upper main sequence, $6 \times 10^{11} M_{\odot}$ of gas have been shed over the entire lifetime of NGC 4472. This corresponds to a mean mass-loss rate of roughly $50 M_{\odot} \mathrm{yr}^{-1}$, some 50 times larger than the rate we have adopted. However, the assumptions underlying the early behavior of galaxies in Tinsley's models, though reasonable, are necessarily somewhat uncertain. It is therefore significant that the more conservative approach adopted here leads to the same uncomfortable contradiction, albeit in a somewhat longer time.

Evidently, the injected gas does not simply collect as neutral hydrogen. In order to examine various alternative assumptions, we state more precisely the minimum conditions which an acceptable solution to the contradiction must satisfy. Elliptical galaxies are observed out to a redshift of at least 0.46 with substantially unchanged colors (Oke 1971; Gunn and Oke 1975). Furthermore, if $q_{0}$ lies between -1 and +1 , the Hubble diagram of Gunn and Oke (1975) implies that galaxy luminosities have varied by less than 30 percent over the look-back time. Luminosities have therefore also remained approximately constant. Constancy of color and luminosity implies a roughly constant mass-loss rate over the look-back time. For $q_{0}=0$ and a Hubble constant of $55 \mathrm{~km} \mathrm{~s}^{-1} \mathrm{Mpc}^{-1}$, the look-back time is $5.5 \times 10^{9} \mathrm{yr}$. The average galaxy in our sample of eight ellipticals has a luminosity of $1.5 \times 10^{10} L_{\odot}$ and a mass-loss rate of $0.22 M_{\odot}$ $\mathrm{yr}^{-1}$. Therefore, the total gas lost over $5.5 \times 10^{9} \mathrm{yr}$ amounts to $1.2 \times 10^{9} M_{\odot}$. The amount shed in NGC 4472, the most luminous object in Paper I, is $6.0 \times 10^{9} M_{\odot}$. (These values exceed the observed upper limits by factors of 6.4 for ellipticals and 30 for NGC 4472.) Hence, a minimum acceptable solution to the contradiction must conceal or remove $1.2 \times 10^{9}$ $M_{\odot}$ of gas in the average E and $6.0 \times 10^{9} M_{\odot}$ of gas in NGC 4472. (These total gas masses correspond to $9.0 \times 10^{8} M_{\odot}$ and $4.5 \times 10^{9} M_{\odot}$ of hydrogen.)

If the mass-loss rate were as much as a factor of 3 smaller (the allowable error estimated in $\S$ II) the contradiction factors given above might be reduced to 2.1 for the average elliptical and 10 for NGC 4472 . It might be argued that the discrepancy would then no longer be significant for the average $\mathrm{E}$, although NGC 4472 would still remain a considerable problem. However, several of our assumptions have acted to minimize the discrepancy. For example, the use of luminosities on the Holmberg system (Paper I) underestimates the total luminosity of the galaxy by neglecting whatever light is present in an extended halo. This might be considerable (Arp and Bertola 1969; de Vaucouleurs 1969; Kormendy and Bahcall 1974). For reasonable choices of the luminosity function, Tinsley's models indicate a brightening of ellipticals over the look-back time. This effect might increase the mass-loss rate by as much as a factor of 1.3-1.4 (Tinsley 1974). Normal elliptical galaxies have been discovered out to redshifts considerably larger than 0.46 (Gunn, private communication), thus increasing the look-back time. Finally, in view of the similarity in morphology between NGC 4472 and other ellipticals, the larger discrepancy for NGC 4472 may well be typical for the class of elliptical galaxies as a whole. For all these reasons, we feel the discrepancy is real, although more stringent upper limits to the $\mathrm{H}$ I masses in E galaxies are clearly desirable.

In the discussion below, possible solutions are divided into two groups. In the first group, termed storage mechanisms, the entire mass of gas is assumed present as a diffuse interstellar medium, but its presence cannot be detected in observations made to date. In the second group, removal mechanisms, the gas disappears from the interstellar medium either by flowing out of the galaxy or by condensing into dense objects-stars, for example. We first consider elliptical galaxies only, reserving our discussion of disk systems for a later section. The discussion below shows that most of the storage "solutions" appear to be impossible on one or more grounds. The present data seem to require the removal of matter from the interstellar medium.

In testing the solutions, we have generally used NGC 3379 as a typical E galaxy, and have assumed that the mass loss is equal to the amount of gas shed in the average elliptical galaxy-namely, $1.2 \times 10^{9} M_{\odot}$. In many cases, one could obtain even more striking contradictions by using NGC 4472, in which the mass to be stored is 5 times larger. However, for testing several of the mechanisms, we require an accurate volume luminosity profile of the test galaxy, and this information is available only for NGC 3379 . We have used Larson's (1974a) model B for the space density distribution of stars in NGC 3379, having adjusted his radial coordinate scale to a distance of $10.0 \mathrm{Mpc}$. Compared with the deconvolution of Miller and Prendergast (1962), Larson's profile has higher densities in the nucleus. However, neither the choice of profile nor the assumed distance of the galaxy affects any of our conclusions.

\section{STORAGE MECHANISMS}

\section{a) Ionized Gas at $T=10,000 \mathrm{~K}$}

Because the ionizing flux from planetary nuclei in elliptical galaxies is quite large (Rose and Tinsley 1974), hydrogen might be photoionized. If it is, and if the heavy element abundance is not too different 
from solar, the gas temperature will be roughly $10,000 \mathrm{~K}$ (Osterbrock 1974).

We first note that the hydrogen cannot be distributed as the stars are. If it were, in order to store $1.2 \times 10^{9} M_{\odot}$ of gas, the central density would be close to $10^{3}$ protons $\mathrm{cm}^{-3}$. At such high densities, the emission measure through the nucleus is $1.4 \times 10^{7}$ $\mathrm{cm}^{-6} \mathrm{pc}$ (corresponding to a seeing disk of $1^{\prime \prime}$ in diameter). This value is 2000 times larger than the emission measure in NGC 4278, an elliptical with striking nuclear emission lines. Such intense emission would be easily detectable.

Evidently, the hydrogen must be distributed more uniformly at lower densities. Suppose then that the gas is distributed at constant density throughout a spherical volume. We have assumed that an emission measure $1 / 20$ that in NGC 4278 would be marginally detectable, corresponding to a value of $400 \mathrm{~cm}^{-6} \mathrm{pc}$. To store $1.2 \times 10^{9} M_{\odot}$ of gas, the sphere radius must exceed $3.3 \mathrm{kpc}$ with a density $\leq 0.25$ protons $\mathrm{cm}^{-3}$.

However, the minimum radius becomes larger if one also considers the balance between recombinations and the ionizing quanta. The principal source of ionizing photons in elliptical galaxies is the nuclei of planetary nebulae. (Since the galaxies are metal-rich, the blue horizontal branch population should be small, and this expectation is confirmed by the lack of strong hydrogen lines in the integrated spectra.) The total emission rate of quanta in NGC 3379 is roughly $2.4 \times 10^{51} \mathrm{~s}^{-1}$. Balancing this against the recombination rate, one finds that the sphere radius must exceed $10 \mathrm{kpc}$.

However, this model seems highly unrealistic on physical grounds. What supports the entire mass of gas in a sphere with such a large radius? A temperature of only $10,000 \mathrm{~K}$ is inadequate to prevent collapse if the gas is to be supported by internal pressure. If the gas is supported in a rotating disk, the mean density and hence the emission measure would be even higher. A disk with a radius larger than the tidal radius of the galaxy would then be required. Thus, there seems to be no plausible way in which the entire mass of gas can be stored in the galaxy at a temperature of 10,000 K.

\section{b) Ionized Gas at $T \approx 4 \times 10^{6} \mathrm{~K}$}

Mathews and Baker (1971) have pointed out that after planetary shells are ejected in elliptical galaxies, they will collide with one another with velocities equal to the relative velocities of the stars. Such collisions would be inelastic, and, if radiative losses during the collisions are unimportant, the resultant temperature of the gas is sufficient to support the gas with a density distribution identical to that of the stars. In an elliptical galaxy having a line-of-sight stellar velocity dispersion of $250 \mathrm{~km} \mathrm{~s}^{-1}$, this temperature is $3.8 \times 10^{6} \mathrm{~K}$.

By applying Larson's stellar density profile to the gas distribution, one can calculate the luminosity in $\mathrm{H} \beta$ and the coronal line $\lambda 5303$ of [Fe XIV] for a model containing $1.2 \times 10^{9} M_{\odot}$ at $3.8 \times 10^{6} \mathrm{~K}$. The surface brightness of $\mathrm{H} \beta$ is $1.1 \times 10^{-14} \mathrm{ergs} \mathrm{cm}^{-2} \mathrm{~s}^{-1} \operatorname{arcsec}^{-2}$ (Gould 1971), corresponding to a seeing disk of $1^{\prime \prime}$ in diameter. At a temperature of $4 \times 10^{6} \mathrm{~K}$, the volume emissivities tabulated by Nussbaumer and Osterbrock (1970) give a surface brightness of $4 \times$ $10^{-13} \mathrm{ergs} \mathrm{s}^{-1} \mathrm{~cm}^{-2} \operatorname{arcsec}^{-2}$ for $\lambda 5303$ of [Fe XIV] if solar abundances are assumed. For comparison, the central surface brightness of NGC 3379 is $2 \times$ $10^{-14} \mathrm{ergs} \mathrm{s}^{-1} \mathrm{~cm}^{-2}$ in a $10 \AA$ bandpass at $5000 \AA$. Hence all of these lines should be visible if the gas were indeed stored at $3.8 \times 10^{6} \mathrm{~K}$. One could reduce these line luminosities by distributing the gas more evenly throughout a larger volume, but then the issue of supporting the gas against collapse would once more become a problem.

\section{c) Dense Cloud Models}

Hydrogen gas could conceivably be hidden in dense clouds in one of two forms - as optically thick neutral hydrogen or as molecular $\mathrm{H}_{2}$. Such clouds might be spherically distributed throughout the galaxy with randomly directed velocities like those of the stars. Alternatively, the clouds might rotate about the nucleus in a disk configuration with circular orbits.

In the first type of model, the clouds cannot be allowed to collide; otherwise, the system of clouds would collapse to a rotating disk, producing a model of the second type. We require that clouds not collide for $10^{9} \mathrm{yr}$ and assume that the clouds are uniformly distributed in a sphere of radius $5 \mathrm{kpc}$ with a line-ofsight velocity dispersion of $250 \mathrm{~km} \mathrm{~s}^{-1}$. If $R_{c}$ is the cloud radius, $n$ is the number of density of clouds, and $v$ is the velocity dispersion, the mean free time of a cloud against collisions is given by (Jeans 1925)

$$
t_{c}=\frac{1}{4 \sqrt{ } 2 \pi R_{c}^{2} n v} \cdot
$$

If $t_{c}>10^{9} \mathrm{yr}$,

$$
N R_{c}^{2}<6.6 \times 10^{4} \mathrm{pc}^{2},
$$

where $N$ is the total number of clouds.

Table 1 summarizes the properties of such clouds for various choices of $N$ and $R_{c} . M$ is the cloud mass, and $n_{\mathrm{H}}$ the neutral hydrogen density inside a cloud. Table 2 describes the characteristics of typical clouds in the solar neighborhood. Compared with local clouds, the clouds in Table 2 are $10^{2}$ to $10^{3}$ times as dense at a given value of the radius. Such dense clouds are hard to stabilize against collapse. In view of the extreme properties of these clouds, together with the fact that virtually all the gas must be located in such clouds, this model seems unlikely.

We therefore abandon the spherically distributed model in favor of a disk configuration. Let the gas be arranged in a uniform disk with thickness $300 \mathrm{pc}$ and radius $5 \mathrm{kpc}$. We characterize the grain-to-gas ratio by the dimensionless parameter $b$, where $b$ is 1 for the solar neighborhood. Suppose first that $b=1$ and that the grains produce a normal reddening law (Whitford 
TABLE 1

Spherically Distributed Cloud Model

\begin{tabular}{cccc}
\hline \hline$N$ & $\begin{array}{c}R_{c} \\
(\mathrm{pc})\end{array}$ & $\begin{array}{c}M_{c} \\
\left(M_{\odot}\right)\end{array}$ & $\begin{array}{c}n_{\mathrm{H}} \\
\left(\mathrm{cm}^{-3}\right)\end{array}$ \\
\hline $7 \times 10^{2} \ldots \ldots \ldots \ldots$ & $<10$ & $1.7 \times 10^{6}$ & $>1.2 \times 10^{4}$ \\
$7 \times 10^{4} \ldots \ldots \ldots \ldots$ & $<1$ & $1.7 \times 10^{4}$ & $>1.2 \times 10^{5}$ \\
$7 \times 10^{6} \ldots \ldots \ldots \ldots$ & $<0.1$ & $1.7 \times 10^{2}$ & $>1.2 \times 10^{6}$ \\
$7 \times 10^{8} \ldots \ldots \ldots \ldots$ & $<0.01$ & 1.7 & $>1.2 \times 10^{7}$ \\
\hline
\end{tabular}

1958). The surface density of neutral hydrogen atoms, $n_{\mathrm{H}}$, is then $1.4 \times 10^{21}$ in the disk, and the optical depth $\tau_{B}$ perpendicular to the disk is 1.1 (Savage and Jenkins 1972; Knapp and Kerr 1974a).

With this large optical depth, such a disk if seen face-on would be easily visible. ${ }^{1}$ If the disk is viewed edge-on, the average optical depth along the line of sight is even larger by a factor of 25 . The edge-on disk will be invisible only if the grain-gas ratio is much smaller (by a factor of $\sim 100$ ) or if the mass is distributed in very small clouds which together cover only a small fraction, say 10 percent, of the area of the edge-on disk. This requirement leads to the condition

$$
N R_{c}{ }^{2}<10^{5} \mathrm{pc}^{2} .
$$

This cloud system is nearly as extreme as that found previously for the spherical case. Therefore, if $b$ is normal, extremely dense clouds are required whether or not clouds are distributed spherically or in a rotating disk.

Let us summarize the results of this discussion. We think it unlikely that all the gas could be located exclusively in dense clouds of the kind implied by equations (1) or (2). If only a few percent of the gas were not in dense clouds, then the matter could be ionized by central stars of planetary nebulae and extensive emission would likely be observable. For this reason, the spherically distributed model and the rotating disk with normal $b$ seem ruled out. Only one model remains, the rotating disk with $b \leqslant 0.01$. Since the integrated absorption-line strengths in elliptical galaxies indicate metal abundances that equal or exceed the solar value (Faber 1973), such a low value

${ }^{1}$ An example of such a disk may exist in NGC 4594. The obvious presence of a large amount of dust coupled with a lack of $\mathrm{H}$ I (Paper I) suggests that the bulk of interstellar gas is in molecular form.

TABLE 2

Clouds in the Solar Neighborhood

\begin{tabular}{|c|c|c|}
\hline $\begin{array}{c}R_{\mathrm{c}} \\
(\mathrm{pc})\end{array}$ & $\begin{array}{c}M_{c} \\
\left(M_{\odot}\right)\end{array}$ & $\begin{array}{c}n_{\mathrm{H}} \\
\left(\mathrm{cm}^{-3}\right)\end{array}$ \\
\hline $\begin{array}{r}20 * \ldots \ldots \ldots \ldots \ldots \ldots \\
3 \dagger \ldots \ldots \ldots \ldots \ldots \\
0.25^{*} \ldots \ldots \ldots \ldots \ldots \\
0.03^{*} \ldots \ldots \ldots \ldots \ldots\end{array}$ & $\begin{array}{c}1.8 \times 10^{4} \\
8 \times 10^{2} \\
3 \\
>0.1\end{array}$ & $\begin{array}{r}2 \times 10 \\
4.4 \times 10^{2} \\
1.6 \times 10^{3} \\
>4 \times 10^{4}\end{array}$ \\
\hline
\end{tabular}

* Spitzer 1968.

$\dagger$ Hollenbach et al. 1971. of $b$ would be at variance with the high metal abundance of the stars. ${ }^{2}$ Even if $b$ is small, both the $\mathrm{H}_{2}$ and the optically thick $\mathrm{H}$ I models still require that virtually all the gas be located in dense clouds (though not necessarily as dense as those found in eqs. [1] and [2]). Furthermore, it would be difficult to form $\mathrm{H}_{2}$ even in dense clouds because the grains necessary to screen out dissociating radiation are lacking when $b$ is small. Though none of these arguments individually is airtight, taken together they render the dense cloud model rather implausible.

If the grain-to-gas ratio in ellipticals is indeed normal, the observed lack of opaque clouds sets a fairly low upper limit on the maximum amount of gas which can be present. The average optical depth of an edge-on gaseous disk containing enough gas to solve the storage problem was 25 . If such a disk is to be invisible with $b=1$, the maximum amount of gas must be roughly two orders of magnitude less, i.e., less than $\sim 10^{7} M_{\odot}$. This result suggests that the actual amount of gas in ellipticals may be a full order of magnitude less than the present upper limits. As long as $b$ is normal, the presence of dark clouds or reddening is a more sensitive indicator of $\mathrm{H} I$ than is the $21 \mathrm{~cm}$ signal.

\section{d) Hydrogen at $2.7 \mathrm{~K}$}

The $21 \mathrm{~cm}$ emission from galaxies is seen against the $2.7 \mathrm{~K}$ blackbody radiation. For an optically thin source, the net brightness temperature measured is $\tau\left(T_{\mathrm{sp}}-T_{\mathrm{BB}}\right)$, where $T_{\mathrm{sp}}$ is the spin temperature of the hydrogen and $T_{\mathrm{BB}}$ is the temperature of the background blackbody spectrum. Therefore, if the temperature of the hydrogen were below $3.2 \mathrm{~K}$ one could store a total of $1.2 \times 10^{9} M_{\odot}$ of gas without violating the observed upper limit of $1.4 \times 10^{8} M_{\odot}$ of hydrogen.

However, it is unlikely that the gas could remain at such low temperatures. Planetary nebulae and other gas clouds shed from stars collide with one another, transforming their kinetic energy of motion into heat. The energy available to heat the gas from this source alone is $9.0 \times 10^{39} \mathrm{ergs} \mathrm{s}^{-1}$. At $3.2 \mathrm{~K}, 21 \mathrm{~cm}$ emission is the principal cooling mechanism, and the total luminosity of $1.2 \times 10^{9} M_{\odot}$ of gas in $21 \mathrm{~cm}$ is only $3.4 \times 10^{34} \mathrm{ergs} \mathrm{s}^{-1}$. Since the energy input to the gas is over five orders of magnitude larger than the energy lost by radiation, it is difficult to see how the gas could stay at temperatures as low as $3.2 \mathrm{~K}$.

\section{REMOVAL MECHANISMS}

Because there appears to be no easy scheme for hiding the required $1.2 \times 10^{9} M_{\odot}$ of gas, we conclude that one or more mechanisms removes the gas from the interstellar medium. Among the various possibilities, we favor the existence of hot galactic winds. However, other processes cannot, from present data, be completely discounted.

\footnotetext{
${ }^{2}$ However, the galaxy NGC 5102 (Paper I) may be an object where $b$ is small. This galaxy has a sizeable mass fraction in $\mathrm{H}_{\mathrm{I}}$ yet no visible dust lanes.
} 


\section{a) Star Formation}

Larson and Tinsley (1974) have presented models of elliptical galaxies in which star formation continues at a rate adequate to consume all the gas currently shed from dying stars. Although such star formation is compatible with the observed integrated colors of elliptical galaxies, the model colors become markedly bluer toward the nuclei. They point out that this behavior is in disagreement with the observed color profiles of elliptical galaxies, most of which are redder near the nuclei. The infrared measurements of $2.3 \mu \mathrm{CO}$ band strengths in early-type galaxies by Frogel et al. (1975) are also consistent with an old stellar population whose light is dominated by $K$ and $\mathbf{M}$ giants. Thus it is improbable that massive, young stars are present but not detectable due, for example, to unusual circumstellar extinction.

However, the model calculations assume an initial mass function fairly rich in hot stars. Since we have no a priori knowledge of the initial mass function in elliptical galaxies, star formation might conceivably be confined to stars of small mass and low luminosity. With this uncertainty, it is not presently possible to place any firm observational limit on the admissible formation rate for low-mass stars. Such a process would, however, be very different from other observed instances of star formation, since there are no signs of dense, dusty clouds, $\mathrm{H}$ il regions, etc. The gas must also be consumed with extreme efficiency. Furthermore, the observations in Paper I indicate that when $\mathbf{H}_{\mathbf{I}}$ is unambiguously present in early-type galaxies, young stars are, in most cases, also present. None of these arguments rule out star formation as a sink for gas, but they also provide no compelling reasons for believing continuous star formation must be occurring in normal elliptical galaxies.

Suppose on the other hand that star formation occurs in bursts which are triggered when the gas mass reaches some critical value. Between bursts the galaxy is quiescent and looks normal. This suggestion has been advanced by van den Bergh (1972) for elliptical galaxies and has been discussed by Biermann and Tinsley (1975) for S0's. This hypothesis, however, cannot explain the lack of gas in the majority of E galaxies because the time scale for burst decay is too long relative to the interval between bursts necessary to keep the gas mass below the detectable limit. A lower limit to burst decay time is provided by the observation that hydrogen absorption lines are strong in the spectra of the galaxies discussed by van den Bergh. Much of the burst light therefore comes from A0 stars, which have a main-sequence lifetime equal to or greater than $3 \times 10^{8}$ yr (Iben 1967). Bursts should be visible for at least this long. Yet for NGC 4472, bursts must occur at least every $2 \times 10^{8} \mathrm{yr}$ (§ III). Therefore, if the outbursts described by van den Bergh are typical, outbursts will overlap in time. Hence virtually all giant ellipticals should display evidence of recent star formation, which they do not.

In view of these objections, it seems unlikely to us that normal star formation, either uniform or non- uniform with time, is the mechanism which removes gas from the majority of elliptical galaxies. However, it will be difficult to rule out completely star formation with low-mass stars. Such a decision would be based on theoretical considerations, and at present the theory of star formation is too rudimentary to allow such a choice.

\section{b) Sweeping by Explosions from Active Nuclei}

According to this suggestion, the nuclei of spheroidal galaxies are prone to recurrent explosions of sufficient violence that the entire galaxy is swept clean of interstellar material. These explosions must occur at least every $10^{9} \mathrm{yr}$ for the average $\mathrm{E}$ galaxy in our sample and every $2 \times 10^{8}$ yr in NGC 4472 .

This suggestion seems unlikely in view of the known positive correlation between radio activity and intrinsic luminosity for elliptical and S0 galaxies (Ekers and Ekers 1973; Colla et al. 1975). The former authors find no compact or extended radio sources in galaxies with $M_{\mathrm{pg}}>-18.5$. Neither is there any optical evidence for violent activity in ellipticals fainter than -18.5 . The optical morphology of elliptical galaxies, however, suggests that most ellipticals fainter than -18.5 also lack $\mathbf{H}$ I. Since large and small ellipticals form a continuous sequence, it is most natural to conclude that all ellipticals are gas poor for the same reasons. Because there is no evidence for violent radio or optical activity in intrinsically faint ellipticals, the explosion mechanism seems unlikely when applied to the class as a whole.

\section{c) Sweeping by Collisions with External Galaxies or Gas Clouds}

From counts of galaxies in clusters, Oemler (1974) has concluded that elliptical galaxies are more common in dense clusters of galaxies than in the general field. This overpreponderance in dense clusters might suggest that collisions with external galaxies, gas clouds, or an intracluster medium can remove gas from a galaxy. This mechanism is akin to the more familiar hypothesis which accounts for S0's in clusters as stripped spirals (Baade and Spitzer 1951; Gunn and Gott 1972).

Though possibly applicable to cluster ellipticals, this theory cannot explain the gas-free nature of ellipticals in the field or in small groups, where peculiar velocities with respect to the Hubble flow are presumably small and where the probability of a collision is remote, at least at the present time. Although field ellipticals might conceivably have resulted through collisional interaction at early epochs, this process by itself cannot explain their gasfree nature today, since sweeping on the order of every $10^{8}-10^{9} \mathrm{yr}$ is required. Furthermore, sweeping by collisions does not account for the clear relation between morphological properties (spheroidal mass distribution, large velocity dispersion, etc.) and low gas content. Put another way, this theory cannot 
explain why virtually every elliptical galaxy has undergone repeated collisions, yet most disk systems have not.

We argue below ( $\S \mathrm{VI})$ that the bulges of spirals are similar to ellipticals in many respects: light distribution, kinematic properties, and stellar population. They also resemble ellipticals in being gas poor (relative to the surrounding disk). In view of these similarities, we speculate that the same mechanism which removes gas in elliptical galaxies is also active in the bulges of spirals. If this conjecture is valid, it provides additional evidence against external collisions because such collisions cannot remove gas from the bulges of spirals while leaving the disks unaffected. Though somewhat speculative, this point is another argument against sweeping by collisions with external objects.

\section{d) Hot Galactic Winds}

In two papers published nearly concurrently, the hot galactic wind hypothesis was described by Johnson and Axford (1970) but explored in more detail in a fundamental paper by Mathews and Baker (1971) (hereafter referred to as MB). Such a wind operates as follows: Because of the large random stellar velocities in spheroidal galaxies, gas clouds shed from dying stars collide violently with one another. The resultant temperature of the interstellar medium is so high that the gas cannot cool efficiently. It remains in this hot, tenuous state and is further heated to higher temperatures by the passage of supernovae shock fronts. Provided that the supernovae rate is sufficiently large, the equilibrium temperature of the gas is so high that the gas is not gravitationally bound to the galaxy and flows out as a hot wind.

The existence of such a wind is based on two requirements. First, the radiative cooling time of the medium must be longer than the time necessary to flow out of the central regions. Otherwise, the material cools and clumps before escaping from the galaxy core, and a thermally unsteady wind results. Whether this occurs depends on the supernovae energy input rate relative to the gas injection rate. The parameter values which result in a thermally steady wind are illustrated by MB in their Figure 4.

The gas injection rate used in this paper is 0.015 $M_{\odot} \mathrm{yr}^{-1}\left(10^{9} L_{\odot}\right)^{-1}$. In the notation of $\mathrm{MB}$, this corresponds to a specific gas injection rate $\alpha_{s}=5 \times 10^{-20}$ $\mathrm{s}^{-1}$ if $M / L_{\mathrm{pg}}=10$. Tammann's (1974) redetermination of the frequency of Type I supernovae yields $2.5 \times$ $10^{-4}$ supernovae per year in an E galaxy with $10^{9} L_{\odot}$. Minkowski (1967) estimates the total energy released in a supernovae of Type $I$ at $10^{51}$ ergs. These two values, expressed in MB's notation, result in a specific energy injection rate $\alpha_{\mathrm{SN}} T_{\mathrm{SN}}$ of $1.6 \times 10^{-12} \mathrm{~K} \mathrm{~s}^{-1}$. Comparison with Figure 4 of MB shows that the predicted winds are well within the thermally steady region. [In a similar calculation, Larson (1974b) has reached the same conclusion.]

The second requirement for a galactic wind relates to the total energy available from supernova heating. This energy must be sufficient to remove all wind particles to infinity as well as to supply all radiative losses before the gas escapes. As shown in $\S \mathrm{V}$, this latter loss is small in comparison with the first requirement and can be ignored. Removing the gas to infinity requires at least an approximate doubling of the kinetic energy per particle. In MB's notation, the required energy is therefore $3 / 2 k T_{s}$, where $T_{s}$ is the temperature corresponding to the stellar velocity dispersion. Thus $T_{s}=v_{s}{ }^{2} m_{\mathrm{H}} / 6 k$, where $v_{s}{ }^{2}$ is the rms stellar velocity dispersion and $m_{\mathrm{H}}$ is the proton mass. Balancing this energy requirement against the energy available from supernovae, one finds that to support a galactic wind,

$$
\alpha_{\mathrm{SN}} T_{\mathrm{SN}} \geq \alpha_{s} T_{s}
$$

The previous values for $\alpha_{\mathrm{SN}} T_{\mathrm{SN}}$ and $\alpha_{s}$ yield $T_{s} \leq$ $3.2 \times 10^{7} \mathrm{~K}$ or $v_{s} \leq 1260 \mathrm{~km} \mathrm{~s}^{-1}$.

This corresponds to a line-of-sight velocity dispersion of $v_{s} / \sqrt{ } 3$, or $730 \mathrm{~km} \mathrm{~s}^{-1}$. Thus all elliptical galaxies with mean line-of-sight velocity dispersions less than $730 \mathrm{~km} \mathrm{~s}^{-1}$ should support a galactic wind.

This limit to $v_{s}$ exceeds the largest velocity dispersions actually observed in giant $\mathrm{E}$ galaxies by about $350 \mathrm{~km} \mathrm{~s}^{-1}$ (Faber and Jackson 1975). However, in view of the uncertainties in the estimate, it is more realistic to conclude that galaxies with the highest velocity dispersions may in fact have difficulty in driving a wind. Recent measurements by Faber and Jackson (1975) show that velocity dispersions increase monotonically with luminosity for elliptical galaxies. Hence the most luminous ellipticals are most likely to retain their gas. In these massive systems, gas which cannot escape may ultimately cool and collect near the nucleus, where it can fuel nuclear radio sources, give rise to emission lines (Disney and Cromwell 1971), and trigger recent star formation (van den Bergh 1972). Thus the wind theory may account in a natural way for the positive correlation between the probability of radio emission and increasing galaxy luminosity among elliptical galaxies (Ekers and Ekers 1973; Colla et al. 1975).

At the other extreme, the wind hypothesis may break down at small values of $v_{s}$. Fundamental to the calculations of MB was the assumption that the equivalent thermal energy of supernovae is deposited uniformly throughout the gas. This they called the assumption of "microscopic evenness." In order for this condition to hold, the mean interval of time between passages of a supernova blast wave must be shorter than the radiative cooling time for the gas, $t_{\text {cool }}$. In their notation,

$$
t_{\mathrm{cool}}=\frac{3 k T_{s}}{n_{e}\left|H\left(T_{s}\right)\right|}
$$

where $H\left(T_{s}\right)$ is the rate coefficient for radiative heating in ergs cm${ }^{-3} \mathrm{~s}^{-1}$, and $n_{e}$ is the electron density. According to the calculations of $H\left(T_{s}\right)$ by Cox and Daltabuit (1971), $T_{s} /\left|H\left(T_{s}\right)\right|$ reaches a minimum near $10^{5} \mathrm{~K}$, corresponding to a total stellar space velocity dispersion of $70 \mathrm{~km} \mathrm{~s}^{-1}$. From the relation between $v_{s}$ and galaxy luminosity found by Faber and Jackson 
(1975), this is appropriate to a galaxy with $M_{B} \approx-15$.

Though a precise calculation of $t_{\text {cool }}$ is difficult, since it is not known how $n_{e}$ scales with galaxy luminosity, it seems possible that in galaxies with $M_{B}$ near -15 , the planetary shells cool and clump before being heated by the passage of a supernova shock. Clouds of gas and dust might then form, together with young stars. In ellipticals of even smaller masses, however, the gas is so loosely bound that almost any disruptive event, such as a single supernova explosion, can sweep the galaxy clean.

This rather simple reasoning suggests, then, a fairly complicated dependence of wind properties on galaxy mass. The least massive dwarf spheroidal systems should be gas free. Near absolute magnitude $M_{B}=$ -15 , one should find a fairly narrow range of luminosities where gas, young stars, and dust can be detected. Above this range most ellipticals should be gas free once more until the most massive systems are encountered. The elliptical galaxies in the Local Group furnish some evidence in favor of these speculations. Two objects, NGC 205 and NGC 185, show evidence of dust clouds and recent star formation (Hodge 1971). The absolute magnitudes of these objects are both close to - 15-being - 15.7 and -14.5 , respectively (van den Bergh 1968). In contrast, the Local Group ellipticals more and less luminous than these two galaxies show no trace of gas or young stars (Hodge 1971).

Yahil and Ostriker (1973) have suggested that mass loss due to galactic winds is responsible for X-ray emission inside clusters of galaxies. The mass shed in winds collects in the cluster, where it is heated to escape velocity by a variety of possible mechanisms. To duplicate the observed X-ray flux for Coma, their published models require gas injection rates in reasonable agreement with the rates used in this paper. Moreover, the injection rate can be varied over wide limits if accompanied by suitable compensating adjustments in other model parameters. Therefore, the wind mechanism as presented here appears to be compatible with observed cluster X-ray sources.

Lastly, we mention recent work by Larson (1974b) on galactic winds during the early history of elliptical galaxies. Based on models of collapsing galaxies (Larson 1974a; Larson and Tinsley 1974), he concludes that winds will become established early in the lifetimes of ellipticals while they are still collapsing. He finds that small ellipticals lose a greater fraction of their mass and, as a result, have lower mean metal abundances and less pronounced central condensations than more massive ellipticals, a prediction in agreement with the observations. His computed winds persist to the present epoch. The agreement he finds between the predicted and observed metal abundances and luminosity profiles for ellipticals may provide further evidence in favor of the existence of galactic winds in spheroidal systems.

\section{e) Summary of Removal Mechanisms}

Among the removal mechanisms, only the hypotheses of star formation and galactic winds are not excluded by the data. Broadly speaking, the wind operates if and only if the stellar velocity dispersion is high, and it is precisely such systems that are deficient in gas, including the nuclear bulges of spirals ( $\S \mathrm{VI})$. The hypothesis of efficient star formation, on the other hand, cannot yet be shown to be related to a galaxy's dynamic structure and is presently unsupported by any direct observational evidence. For these reasons it seems less attractive at the present time. In the remainder of this paper we examine the wind hypothesis in more detail and speculate that winds might exist in spiral bulges as well as in elliptical galaxies.

\section{DUST IN ELLIPTICAL GALAXIES}

Infrared excesses in planetary nebulae and $M$ giants indicate that substantial amounts of dust should be shed along with the gas in elliptical galaxies. Therefore, we expect that dust as well as gas is injected into the interstellar medium in these objects. This dust will move along with the general flow. Its presence might have two potentially significant consequences which should be evaluated. Through reddening and absorption of starlight it influences the color and luminosity profiles of the galaxy. Yet the observational evidence indicates that internal reddening in $\mathrm{E}$ galaxies is small. We have tested whether the predicted grain optical depth in winds is consistent with observed upper limits to the reddening. For a normal grain-togas ratio (Savage and Jenkins 1972; Knapp and Kerr 1974a), we find for our model based on NGC 3379 a total visual optical depth $\tau_{v} \leqslant 0.02$. Such a small effect would not be detectable.

Second, grain-gas collisions might be important by cooling the gas more efficiently than does thermal bremsstrahlung alone. This process might conceivably make a hot wind impossible. Yahil and Ostriker (1973) have pointed out that grain-gas cooling cannot be significant because the grains are destroyed by sputtering roughly 10 times faster than they can cool the gas. Hence the wind will survive despite the injection of grains into the flow. On the other hand, the sputtering rate is probably not fast enough to destroy the grains before they leave the galaxy. Using sputtering times from Burke and Silk (1974), and comparing with characteristic flow times for the wind in NGC $3379(\mathrm{MB})$, we find that $t_{\mathrm{gr}}$ is larger than $t_{\text {flow }}$ by roughly an order of magnitude. Hence it seems probable that grains survive the wind largely intact, but their computed reddening effect is well below observable limits.

\section{WINDS IN SPIRAL GALAXIES}

In the previous sections we have discussed evidence for the existence of winds or some other removal mechanism in elliptical galaxies. This section briefly outlines additional reasoning suggesting that if such processes occur in ellipticals, then they may also exist in the bulges of spiral galaxies.

We first review some general parameters which might govern the behavior of wind models. Winds 
should be less efficient in systems where relative velocities in cloud-cloud collisions are small, the gas density is high, or the supernova rate is low. Hence a wind in a pure cold-disk system of stars would probably require either very low density to minimize cooling or a large energy-input rate. In spiral galaxies, the relative amounts of low- and high-velocity stellar populations per unit volume vary strongly as a function of position in the galaxy, as do the mass-loss and supernova rates. If winds exist in spirals, their behavior must be quite complex, since basic wind parameters vary greatly within the galaxy. In view of these difficulties, realistic models of galactic winds in spiral galaxies will not be simple to construct. We have therefore limited the present discussion to a preliminary and necessarily speculative exploration of possible effects from winds in bulges of massive spirals. In concluding this section, we briefly discuss the existence of the S0 galaxies as a class in terms of effects from hot winds or other removal mechanisms.

\section{a) Nuclear Bulges of Spirals}

The bulges of spirals apparently resemble elliptical galaxies in three important respects: ${ }^{3}$ (1) The stellar velocity dispersions are fairly large, as evidenced by observations of stars in the halo of our galaxy. (2) The bulge light is produced by a stellar population very similar to the population in ellipticals (Spinrad 1962; Spinrad and Taylor 1970; Spinrad et al. 1971; van den Bergh 1971). (3) Both optical and radio evidence indicates the nuclear bulges of spirals are gas poor relative to the surrounding disks. Ordinary spiral arms and $\mathrm{H}$ II regions cease at the edge of the bulge region in most spirals (re H II regions, see Lynds 1974; Burton et al. 1975). The intensity of radio emission from $\mathrm{CO}$ declines in the bulge region of the Milky Way (Scoville and Solomon 1975; Burton et al. 1975). This suggests that the bulge is poor in molecules as compared with the disk, since $\mathrm{CO}$ and $\mathrm{H}_{2}$ content may be correlated. $\mathrm{H}$ I surface density maps for 14 galaxies also indicate the central bulges of spirals are deficient in gas. Although it is difficult to compare these maps because of differences in instrumentation or technique, it does appear that the depth and width of the central $\mathrm{H}$ I minimum are correlated with the prominence of the nuclear bulge on optical photographs. In the two $\mathrm{Sb}$ galaxies that have been observed (M31 and M81) the depression is large and deep (e.g., Roberts 1967; Rots and Shane 1974). The intermediate Sc's and Scd's show a dip of moderate strength and size (Rogstad and Shostak 1972; Burton 1973; Huchtmeier 1973; Rogstad et al. 1974). The Irr I's, on the other hand, show a central peak (McGee 1964; Hindmann 1967; Shostak 1974; Shostak and Rogstad 1973a,b).

${ }^{3}$ This is not to say that spiral bulges and ellipticals are identical. The disk gas component, though reduced in density, extends deep into the bulge region. The interaction between the removal mechanism and this gaseous component is probably quite complex and conceivably could account for some of the unusual properties of interstellar matter in the nuclear regions of spirals.
Given these potential similarities in basic physical characteristics, winds in spiral bulges as well as in elliptical galaxies are not without theoretical foundation. However, three other possible explanations might possibly account for the central minima in $\mathrm{H}$ I maps of spirals. First, most of the nuclear gas may be molecular $\mathrm{H}_{2}$, as has been suggested for our own galaxy (e.g., Scoville et al. 1974). The second explanation calls for efficient star formation to deplete the interstellar medium (Pikel'ner 1970; Sandage et al. 1970). A third possibility is that the nucleus is periodically swept by explosive nuclear activity (Sanders and Prendergast 1974). Although there is presently insufficient data to firmly decide on one of these four alternative mechanisms, so far only the wind hypothesis provides a physical basis for the observed correlation between spheroidal stellar populations and low gas content.

If we accept the hypothesized connection between gas deficiency and elliptical-like stellar populations, then those regions of a spiral galaxy dominated by a hot stellar component should tend to be gas poor (as a result of wind sweeping or some other mechanism). The $\mathrm{H}$ I surface maps suggest that these gas-poor regions are roughly coextensive with the optical bulge. Hence the fraction of the galaxy in which spiral-arm type star formation is an important gas sink should decrease as the bulge-to-disk ratio $(B / D)$ increases.

It is well known that $B / D$ and Hubble type are closely related. For example, form types on the Yerkes classification system (Morgan 1958, 1959) can be used as a subjective measurement of $B / D$. De Vaucouleurs (1962) has shown that Yerkes and Hubble types are closely correlated. However, Hubble types are based both on the appearance of the Population I component and more indirectly on $B / D$ (Sandage 1961). Thus two rather different criteria, appearance of Population $\mathrm{I}$ and $B / D$, tend to yield similar results. We speculate that this similarity is an indication of the disruption and reduction of the total $\mathrm{H} \mathrm{I}$ in the disk component as a result of removal mechanisms operating in the bulge. According to this reasoning, spirals with large bulges should contain relatively little gas whereas those with small or nonexistent bulges should be rich in gas. Of course, in presenting these ideas we are aware of the large amount of scatter which exists in all relationships for galaxies, but we feel that our discussion is representative of the broad trend apparent in characteristics of normal galaxies.

\section{b) The SO Problem}

The correlation between $B / D$ and gas content may also provide a means for accounting for the S0 class of galaxies. The importance of S0's for the problem of galaxy formation and evolution has been emphasized by Sandage et al. (1970). Dynamically, S0's resemble spirals, yet they usually possess a stellar population and gas content typical of ellipticals. In terms of several classification criteria, S0's appear to form a transition between spirals and ellipticals. In 
TABLE 3

Properties of S0's Compared with Properties OF OTHER HubBle TyPes

\begin{tabular}{|c|c|c|c|c|}
\hline Type & $\left\langle M_{\mathrm{H} \mathrm{I}} / L_{\mathrm{pg}}\right\rangle^{*}$ & $(B-V)_{0} \dagger$ & $\begin{array}{l}\text { Mean } \\
\text { Yerkes } \\
\text { Typeł }\end{array}$ & $\begin{array}{c}\text { Observed } \\
\text { Frequency } \\
\text { of } \lambda 3727 \S(\%)\end{array}$ \\
\hline $\begin{array}{l}\text { E. } \ldots \ldots \ldots \\
\text { So } \ldots \ldots \ldots \\
\text { Sa....... } \\
\text { Sb. } \\
\text { Sc....... } \\
\text { Sd } \ldots \ldots \\
\text { Irr I..... }\end{array}$ & $\begin{array}{c}\leq 0.01 \| \\
\leq 0.05 \# \\
\leq 0.16^{* *} \\
0.19 \dagger \dagger \\
0.40 \dagger \dagger \\
0.78 \dagger \dagger \\
\ldots\end{array}$ & $\begin{array}{l}0.90 \\
0.88 \\
0.82 \\
0.74 \\
0.54 \\
0.44 \\
0.39\end{array}$ & $\begin{array}{l}7.0 \\
6.8 \\
5.7 \\
4.4 \\
2.8 \\
2.1 \\
1.0\end{array}$ & $\begin{array}{l}15 \\
38 \\
54 \\
73 \\
76 \\
\dddot{94}\end{array}$ \\
\hline
\end{tabular}

* In solar units.

$\dagger$ De Vaucouleurs and de Vaucouleurs 1972. Corrected for galactic reddening and for redshift.

$\ddagger$ Data from Morgan 1959. Expressed numerically on a scale $a=1, k=7$.

$\S$ Osterbrock 1969. Mean of Mt. Wilson and Lick data.

II Mean of 8 E's (Paper I).

\#Mean of all S0 detections and upper limits from Paper I.

** Mean of all Sa detections and upper limits, as given in Fig. 8, Paper I.

$\dagger \dagger$ Means from Fig. 8, Paper I.

Table 3 we have collected some characteristics of galaxies as a function of Hubble type; in the respects enumerated in the table, the S0 class represents a smooth transition between the spirals and ellipticals. Most important, the mean gas content of S0's also appears to be intermediate between spirals and ellipticals. Because of their critical transitional nature, a successful theory for S0's might account for several general characteristics of the Hubble sequence as a whole.

We now propose that S0's are simply gas-deficient spirals (cf. Sandage et al. 1970); most, but not all, normal S0's fit this definition (Paper I). If this deficiency is a result of an internal removal mechanism, then our earlier arguments suggest that the typical S0 should have a large $B / D$ ratio. This conclusion appears to agree with the evidence from Yerkes types, which indicates that as a class, S0's have earlier form types and hence larger bulges than later type spirals. Freeman's (1970) more quantitative data on $B / D$ also show systematically large bulges in S0's, but with considerable scatter. As these data are, in some cases, based on older photographic photometry, the accuracy of the results is somewhat doubtful. It would therefore be desirable to repeat this type of quantitative study using modern methods of surface photometry.

It is well known that S0's are abnormally common in dense clusters of galaxies (e.g., Oemler 1974). This may be evidence for the production of S0's by external stripping during collisions either with other galaxies (Baade and Spitzer 1952) or with intracluster gas clouds (Gunn and Gott 1972). If, however, such stripping is the sole source of the overpreponderance of S0's, the proportion of E's should be fixed-that is, the total number of S0's plus spirals should be
TABLE 4

Hubble Types in Clusters of Galaxies FROM OEMLER (1974)

\begin{tabular}{|c|c|c|c|}
\hline Cluster type & $\mathrm{E}(\%)$ & S0 (\%) & Spiral (\%) \\
\hline 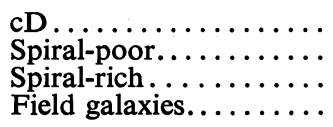 & $\begin{array}{l}36 \\
17 \\
16 \\
13\end{array}$ & $\begin{array}{l}44 \\
55 \\
34 \\
23\end{array}$ & $\begin{array}{l}20 \\
28 \\
50 \\
64\end{array}$ \\
\hline
\end{tabular}

independent of cluster morphology. The average percentage populations of morphological types in Oemler's three kinds of clusters are shown in Table 4. We see that the $\mathrm{cD}$ clusters have a large proportion of ellipticals. This fact suggests that stripping is, after all, not the sole source of S0's in clusters. Furthermore, Turner and Sargent (1974) find that virtually all the local $\mathrm{E}$ galaxies are located in gravitationally bound groups. The data on ellipticals in clusters and in small groups therefore suggest that a large density of nearby companions (in a group or cluster) favors the formation of systems with large bulges. If this conclusion is correct, the greater number of S0's in clusters may not be due to stripping, but may simply be a consequence of the greater number of systems formed with large $B / D$.

Finally, we noted in § III that in the absence of some removal mechanism, detectable amounts of neutral hydrogen should collect in an average S0 in only $\sim 10^{9} \mathrm{yr}$. Therefore, some continuous sweeping mechanism must operate to rid S0's of gas (Biermann and Tinsley 1975 have reached the same conclusion). This fact is evidence against stripping as the sole source of S0's, since collisions must be rare at present for S0's in the field and in the outskirts of clusters. Even if stripping removed gas from S0's at an early epoch, we therefore still require an internal removal mechanism to keep them clean at the present time. If such a process for some reason cannot operate, then it is a provocative possibility that some S0's may evolve in an opposite sense to that usually envisioned-namely, from gas-poor to gas-rich systems.

We mentioned above that the correlations between gas content, Hubble type, and $B / D$ are not exact. This fact was noted by Sandage (1961) and later discussed at length by Sandage et al. (1970). The S0 galaxy NGC 4762 is striking evidence for this conclusion. Figure 1 shows that NGC 4762 has a bulge size typical of an $\mathrm{Sb}$, yet it is a classic $\mathrm{S} 0$ and has a very small $\mathrm{H}$ I fraction (Paper I).

The existence of galaxies like NGC 4762 is extremely important and poses a challenge to our assumed correlation between bulge size and gas removal mechanism. We do not brush such examples aside lightly. However, we can enumerate several factors which might play a role in causing the general scatter between $B / D$, gas content, and Hubble type. Among these are observational errors, gas in the form of molecular $\mathrm{H}_{2}$ (not detected in $21 \mathrm{~cm}$ ), and dependence of Hubble type on density-wave parameters (e.g., Roberts et al. 1975) or disk density (Talbot and Arnett 1975). 

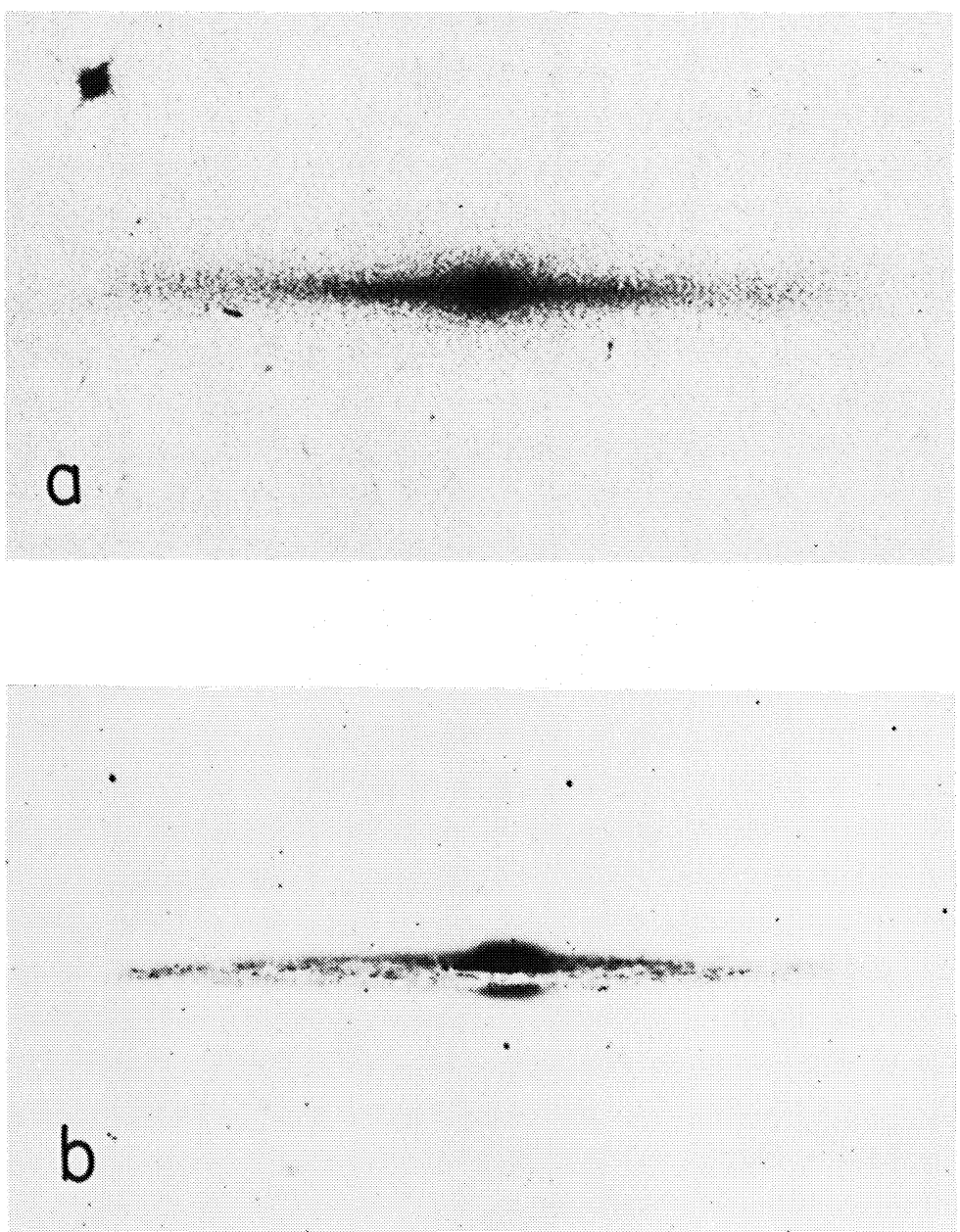

FIG. 1.-(a) NGC 4762, a normal S0 with a small value of $B / D$ (Crossley photo). (b) NGC 4565 , a normal Sb with a value of $B / D$ typical for an $\mathrm{Sb}(120$ inch $[3.0 \mathrm{~m}]$ photo). 
[Page intentionally left blank; back of preceeding plate page.] 
Among the various removal possibilities, the hot wind hypothesis may actually prove consistent with the scatter in $B / D$ since several mechanisms exist which might reduce an otherwise high gas density to a point where a wind could be maintained. These include sweeping by a nuclear explosion, an epoch of strong supernovae activity (Larson 1974b), and collisional stripping. (Perhaps one of these mechanisms is responsible for NGC 4762.) On the other hand, collisional accretion of gas might raise gas content above the average level. Intergalactic clouds of neutral hydrogen are known in small groups of galaxies (Mathewson et al. 1975; Roberts 1972; Gottesman and Weliachew 1975). Masses of these clouds range up to $10^{9} M_{\odot}$, an appreciable fraction of the neutral hydrogen mass in an average galaxy. If a galaxy and gas cloud collided with sufficiently small relative velocity, perhaps a sizeable fraction of the cloud might be accreted. Infall processes similar to this have been discussed by Oort (1970) and others. In short, any or all of these processes of sweeping or accretion may play a role in creating scatter between $B / D$, gas content, and Hubble type.

There exists a comparatively simple observational test to measure the importance of collisional accretion or stripping in determining gas content and Hubble type. This test involves a comparison between $B / D$ for S0's in clusters, small groups, and the general field. If $B / D$ is identical for all objects, whether a galaxy is an S0 evidently depends on only its internal characteristics. If $B / D$ for objects in clusters or small groups is systematically different, this would indicate that intrinsically different galaxies can become S0's depending on the ambient conditions. The same comparison could, of course, be extended to other morphological types as well.

\section{SUMMARY}

In this paper we have discussed the implications of the low upper limits to neutral hydrogen content observed in $\mathrm{E}$ and $\mathrm{S} 0$ galaxies. We reviewed the evidence for mass loss in these systems and adopted a current mass-loss rate based on stellar population models of Larson and Tinsley. If gas shed by dying stars is completely retained by these galaxies and exists as $\mathrm{H} \mathrm{I}$, the upper limits will be exceeded in only $10^{9} \mathrm{yr}$ for the average E and S0 and in only $2 \times 10^{8} \mathrm{yr}$ for NGC 4472. Evidently, gas either is hidden in some undetectable form or is removed from the interstellar medium completely.

Several schemes to conceal the mass were considered, including ionized gas, dense clouds of $\mathrm{H}_{2}$ or optically thick neutral hydrogen, and very cold $\mathrm{H}$ I with a temperature near $3 \mathrm{~K}$. All proposals failed on one or more grounds. Hence a removal mechanism seems likely. Various possibilities include efficient star formation, sweeping by nuclear explosions, and hot, supernovae-driven galactic winds. Of these, we feel the wind hypothesis offers several attractive features, although none of the above mechanisms are totally inconsistent with present data. In addition to explaining the observed lack of $\mathrm{HI}$, winds in ellipticals are apparently in agreement with several other observations, including (1) the lack of observed reddening in early-type galaxies, (2) the existence of young stars and dust in luminous dwarf ellipticals like NGC 205, (3) the fact that radio activity is associated preferentially with massive ellipticals, (4) the observed dependence of metallicity in ellipticals on galaxy mass (Larson 1974b), and (5) the existence of $\mathrm{X}$-ray sources in clusters of galaxies. We purposefully avoided mention of possible interactions between a wind and the external environment. However, for galaxies inside clusters, ram pressure and heating of galactic gas by electron conduction (Gisler 1975; Gun and Gott 1972; de Young 1974) might also influence wind behavior.

The paper closes with a speculative discussion of the possibility of internal removal mechanisms in the bulges of disk galaxies. Spiral bulges have several similarities to ellipticals, including a large stellar velocity dispersion and an old stellar population. We therefore suggest that the same removal mechanisms operating in elliptical galaxies will probably also be present in spiral bulges. Such processes may account for the central minima in $\mathrm{H}$ I maps of spirals with large bulges. Again, the hot wind appears to be an especially attractive explanation, since it provides a direct physical connection between gas content and stellar velocity dispersion. Since the efficiency of such a wind in sweeping gas from a galaxy might plausibly depend on the relative importance of the bulge component, one might expect the overall gas content of disk systems to vary inversely with the bulge-to-disk ratio. This hypothesis seems consistent with several observed properties of the Hubble sequence in general and S0 galaxies in particular.

We would like to thank Drs. W. G. Mathews, B. M. Tinsley, and B. Balick for their advice and comments. We would also like to acknowledge useful suggestions from Drs. J. and L. Goad.

Note added in proof.-As a test for the presence of a molecular interstellar medium in early-type galaxies, we have made a preliminary search for ${ }^{12} \mathrm{CO} 115 \mathrm{GHz}$ emission in a few systems using the NRAO $11 \mathrm{~m}$ telescope on Kitt Peak during 1975 May 26-31. No galaxies were detected. Upper limits of a few tenths of a degree antenna temperature were found for NGC $520,2685,3077$, and 5102. NGC 4594 was also not detected to a level of about $0.1 \mathrm{~K}$.

\section{REFERENCES}

Arp, H. C., and Bertola, F. 1969, Ap. Letters, 4, 23. Baade, W., and Spitzer 1951, Ap. J., 113, 413. Biermann, P., and Tinsley, B. M. 1975, preprint.
Bottinelli, L., Gougenheim, J., and Heidmann, J. 1973, Astr. and $A p ., 25,319$.

Burke, J. R., and Silk, J. 1974, Ap. J., 190, 1. 
Burton, W. B. 1973, Pub. A.S.P., 85, 679.

Burton, W. B., Gordon, M. A., Bania, T. M., and Lockman, F. J. 1975, preprint.

Colla, G., Fanti, C., Fanti, R., Gioia, I., Lani, C., Lequeux, J., Locas, R., and Ulrich, M. H. 1975, preprint.

Cox, D. P., and Daltabuit, E. 1971, Ap. J., 167, 113.

de Vaucouleurs, G. 1962, IAU Symposium No. 15, ed. G. McVittie (New York: Macmillan), p. 11. . 1969, Ap. Letters, 4, 17.

de Vaucouleurs, G., and de Vaucouleurs, A. 1964, Reference Catalogue of Bright Galaxies (Austin: University of Texas Press).

-. 1972, Mem. R.A.S., 77, 1.

de Young, D. 1974, private communication.

Disney, M. J., and Cromwell, R. H. 1971, Ap. J. (Letters), 164, L35.

Ekers, R. D., and Ekers, J. A. 1973, Astr. and Ap., 24, 247.

Faber, S. M. 1972, Astr. and Ap., 20, 361. 1973, Ap. J., 179, 731.

Faber, S. M., and Jackson, R. 1976, Ap. J., in press.

Ford, H. C., and Jenner, D. C. 1975, preprint.

Ford, H. C., Jenner, D. C., and Epps, H. W. 1973, Ap. J. (Letters), 183, L73.

Freeman, K. C. 1970, $A p . J ., 160,811$.

Frogel, J. A., Persson, S. E., Aaronson, M., Becklin, E. E., Mathews, K., and Neugebauer, G. 1975, Ap. J. (Letters), 195, L15.

Gallagher, J. S., III. 1972, A.J., 77, 568.

Gallagher, J. S., III, Faber, S. M., and Balick, B. 1975, Ap. J., 202, 7 (Paper I).

Gisler, G. R. 1975, Bull. AAS, 7, No. 2, Part I, 263.

Gottesman, S. T., and Weliachew, L. 1975, Ap. J., 195, 23.

Gould, R. J. 1971, Ap. Letters, 8, 129.

Gunn, J. E., and Gott, J. R., III. 1972, Ap. J., 176, 1.

Gunn, J. E., and Oke, J. B. 1975, $A p$. J., 195, 255.

Hindmann, J. V. 1967, Australian J. Phys., 20, 147.

Hodge, P. W. 1971, Ann. Rev. Astr. and Ap., 9, 35.

Hollenbach, D., Werner, M. W., and Salpeter, E. E. 1971, Ap. J., 163, 165.

Huchtmeier, W. 1973, Astr. and Ap., 9, 35.

Iben, I., Jr. 1967, Ann. Rev. Astr. and Ap., 5, 571.

Jeans, J. H. 1925, The Dynamical Theory of Gases (4th ed.; Cambridge: Cambridge University Press), p. 37.

Johnson, H. E., and Axford, W. I. 1971, Ap. J., 165, 381.

Knapp, G. R., and Kerr, F. J. 1974a, Astr. and Ap., 35, 361. $1974 b, A . J ., 79,667$.

Kormendy, J., and Bahcall, J. N. 1974, A.J., 79, 671.

Larson, R. B. $1974 a$, M.N.R.A.S., 166, 585. $1974 b$, ibid., 169, 229.

Larson, R. B., and Tinsley, B. M. 1974, Ap. J., 192, 293.

Lynds, B. T. 1974, Ap. J. Suppl., No. 267, 28, 391

Mathews, W. G., and Baker, J. C. 1971, Ap. J., 170, 241 (MB).

Mathewson, D. S., Cleary, M. N., and Murray, J. D. 1975, Ap. J. (Letters), 195, L97.

McGee, R. X. 1964, Australian Journal of Phys., 17, 515.

Miller, R. H., and Prendergast, K. H. 1962, Ap. J., 136, 713

Minkowski, R. 1967, in Stars and Stellar Systems, Vol. 7, ed. L. H. Aller and B. M. Middlehurst (Chicago: University of Chicago Press), chap. 11.
Morgan, W. W. 1958, Pub. A.S.P., 70, 364. . 1959, ibid., 71, 394.

Nussbaumer, H., and Osterbrock, D. E. 1970, Ap.J., 161, 811. Oemler, A. E. 1974, Ap. J., 194, 1.

Oke, J. B. 1971, $A p . J ., 170,193$.

Oort, J. H. 1970, Astr. and Ap., $2,381$.

Osterbrock, D. E. 1969, Forbidden Transitions in Stellar Spectra: Liège Colloquium No. 15 (Liege: University of Liege), p. 39.

Freeman)

Pikel'ner, S. B. 1968, Ann. Rev. Astr. and Ap., 6, 165.

Quirk, W. J., and Tinsley, B. M. 1973, Ap. J., 179, 69.

Roberts, M. S. 1967, in IAU Symposium No. 44, ed. H. van Woerden (London: Academic Press).

-. 1969, A.J., 74, 859.

1972, in External Galaxies and Quasi-Stellar Objects, ed. D. S. Evans (Dordrecht: Reidel), p. 12.

Roberts, W. W., Roberts, M. S., and Shu, F. 1975, Ap. J., 196, 381

Rogstad, D. H., Lockhart, I. A., and Wright, M. C. H. 1974, Ap. J., 193, 309.

Rogstad, D. H., and Shostak, G. S. 1972, Ap. J., 176, 315.

Rose, W. K., and Tinsley, B. M. 1974, Ap. J., 190, 243.

Rots, A. H., and Shane, W. W. 1974, Astr. and Ap., 31, 245.

Sandage, A. 1961, The Hubble Atlas of Galaxies (Washington: Carnegie Institution of Washington).

Sandage, A., Freeman, K. C., and Stokes, N. R. 1970, Ap. J., 160, 831 .

Sanders, R. H., and Prendergast, K. H. 1974, Ap. J., 188, 489.

Savage, B. D., and Jenkins, E. B. 1972, Ap. J., 172, 491.

Scoville, N. Z., and Solomon, P. M. 1975, Ap. J. (Letters), 199, L105.

Scoville, N. Z., Solomon, P. M., and Jefferts, K. B. 1974, Ap. J. (Letters), 187, L63.

Shostak, G. S. 1974, Astr. and Ap., 31, 97.

Shostak, G. S., and Rogstad, D. H. 1973a, Astr. and Ap., 24, 405.

S. 1973b, ibid., 24, 411.

Spinrad, H. 1962, Ap. J., 135, 715.

Spinrad, H., Gunn, J. E., Taylor, B. J., McClure, R. D., and Young, J. W. 1971, Ap. J., 165, 11.

Spinrad, H., and Taylor, B. J. 1970, Ap. J. Suppl., No. 193, $22,445$.

Spitzer, L. 1968, Diffuse Matter in Space (New York: Interscience), p. 81 .

Talbot, R., and Arnett, W. D. 1975, Ap: J., 197, 551.

Tammann, G. A. 1974, in Supernovae and Supernovae Remnants, ed. C. B. Cosmovici (Dordrecht: Reidel).

Tinsley, B. M. 1973, Ap. J., 186, 35.

1974, Synthesis of Stellar Populations, lecture for Scuola Superiore di Astronomia, Erice, Sicily, 1974 May.

Turner, E. L., and Sargent, W. L. W. 1974, Ap. J., 194, 587. van den Bergh, S. 1968, J.R.A.S. Canada, 62, 145.

-. 1971, A.J., 76, 1082.

- 1972, J.R.A.S. Canada, 66, 237.

Weidemann, V. 1968, Ann. Rev. Astr. and Ap., 6, 351

Whitford, A. E. 1958, A.J., 63, 201.

Yahil, A., and Ostriker, J. P. 1973, Ap. J., 185, 787.

S. M. FABER: Lick Observatory, University of California, Santa Cruz, CA 95064

J. S. Gallagher: School of Physics and Astronomy, University of Minnesota, Minneapolis, MN 55455 